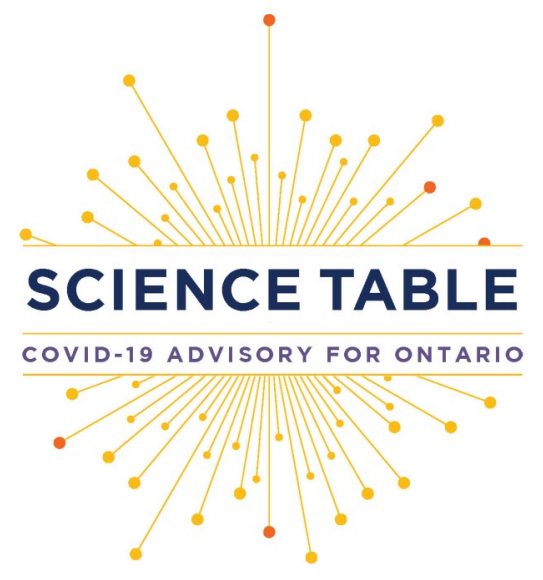

Version 2.0

Published: May 7, 2021

Version 1.0 is available under Additional Resources at https://doi.org/10.47326/ ocsat.2021.02.16.2.0

Citation: Pai M, Chan B, Stall NM, et al. Vaccine-induced immune thrombotic thrombocytopenia (VITT) following adenovirus vector COVID-19 vaccination: lay summary. Science Briefs of the Ontario COVID19 Science Advisory Table. 2021;2(16). https:// doi.org/10.47326/ocsat.2021.02.16.2.0

Author Affiliations: The affiliations of the members of the Ontario COVID-19 Science Advisory Table can be found at https:// covid19-sciencetable.ca/.

Declarations of Interest: The declarations of interest of the members of the Ontario COVID-19 Science Advisory Table, its Working Groups, or its partners can be found at https:// covid19-sciencetable.ca/. The declarations of interest of external authors can be found under additional resources at https:// doi.org/10.47326/ocsat.2021.02.16.2.0.

About Us: The Ontario COVID-19 Science Advisory Table is a group of scientific experts and health system leaders who evaluate and report on emerging evidence relevant to the COVID-19 pandemic, to inform Ontario's response. Our mandate is to provide weekly summaries of relevant scientific evidence for the COVID-19 Health Coordination Table of the Province of Ontario, integrating information from existing scientific tables, Ontario's universities and agencies, and the best global evidence. The Science Table summarizes its findings for the Health Coordination Table and the public in Science Briefs.

The Drugs \& Biologics Clinical Practice Guidelines Working Group is a group of clinicians and scientists with recognized expertise in drugs, biologics, and clinical care. The Working Group will evaluate existing scientific data, disease epidemiology, drug availability, and implementation issues in order to develop Clinical Practice Guidelines for the treatment of COVID-19 using drugs and biologics. The Working Group reports its findings to the public and the Science Table. Its findings are

\section{Vaccine-Induced Immune Thrombotic Thrombocytopenia (VITT) Following Adenovirus Vector COVID-19 Vaccination}

Menaka Pai, Benjamin Chan, Nathan M. Stall, Allan Grill, Noah Ivers, Antonina Maltsev, Katherine J. Miller, Ayodele Odutayo, Fahad Razak, Michael Schull, Brian Schwartz, Michelle Sholzberg, Robert Steiner, Sarah Wilson, Ullanda Niel, Peter Jüni, Andrew M. Morris on behalf of the Drugs \& Biologics Clinical Practice Guidelines Working Group and the Ontario COVID-19 Science Advisory Table

\section{Lay Summary}

\section{What do we know so far?}

The AstraZeneca/COVISHIELD COVID-19 vaccine appears to be associated with rare cases of serious blood clots, including blood clots in the brain and other critical organs. Similar cases have also been reported following the Janssen/Johnson \& Johnson COVID-19 vaccine. These blood clots have two important features: they can occur 4 to 28 days after vaccination, and they are associated with low platelets (tiny blood cells that help form blood clots to stop bleeding). Doctors are calling this "vaccine-induced immune thrombotic thrombocytopenia" (VITT), "vaccine-induced prothrombotic immune thrombocytopenia" (VIPIT), or "thrombotic thrombocytopenic syndrome" (TTS). These blood clots are different from the blood clots most people are familiar with; they are more aggressive and are more likely to cause death or severe disability, even with early diagnosis and treatment.

Published estimates of the incidence of VITT range from 1 case per 26,000 to 1 case per 127,000 doses of AstraZeneca/COVISHIELD administered. The estimates vary by country, with countries like Norway and Denmark reporting the highest rates. As of April 28, 2021, the rate of VITT in Canada has been estimated to be approximately 1 per 100,000 doses. Nearly all reported cases have occurred after the first dose. There have been few reported cases of VITT with the Janssen/Johnson \& Johnson vaccine, so it is difficult to calculate a precise rate, but the incidence of VITT appears to be approximately 1 case per 500,000 vaccine doses administered.

\section{Are certain people more likely to get VITT?}

At this time, we do not know if certain patients are more likely to get VITT. Currently, we do not have any evidence that VITT is more common in people who have had blood clots before, people with a family history of blood clots, people on birth control or other hormones, people with autoimmune disease, people with low platelets or other platelet disorders, or pregnant people, because VITT does not develop through the same process as more common types of bleeding or clotting problems. It may be possible, however, that people with a history of heparin induced thrombocytopenia (HIT) or cerebral sinus vein thrombosis (CSVT) with low platelets are at increased risk of VITT; they should receive an mRNA vaccine (Pfizer or Moderna) rather than an adenoviral vector vaccine. 
What should you look out for if you received the AstraZeneca/COVISHIELD or Janssen/Johnson \& Johnson COVID-19 vaccine? Ontario COVID-19 Science Advisory Table (info@covid19-sciencetable.ca)

Copyright: 2021 Ontario COVID-19 Science Advisory Table. This is an open access document distributed under the terms of the Creative Commons Attribution License, which permits unrestricted use, distribution, and reproduction in any medium, provided that the original work is properly cited.

The views and findings expressed in this Science Brief are those of the authors and do not necessarily reflect the views of all of the members of the Ontario COVID-19 Science Advisory Table, its Working Groups, and its partners.

You should speak to a health care professional if you have unusual or severe symptoms after any COVID-19 vaccine. If you experience the following symptoms that start between 4 and 28 days after vaccination, it might indicate that you have VITT: a severe headache that does not go away; a seizure; difficulty moving part of your body; new blurry vision or double vision that does not go away; difficulty speaking; shortness of breath; severe chest, back, or abdominal pain; unusual bleeding or bruising; new reddish or purplish spots, or blood blisters; or new severe swelling, pain, or colour change of an arm or a leg. These symptoms can also be a sign of other serious conditions and should be assessed in an emergency department.

\section{What should you do if you have concerning symptoms after the AstraZeneca/ COVISHIELD or Janssen/Johnson \& Johnson COVID-19 vaccine?}

If you have severe symptoms such as those listed above, you should go to the nearest emergency department immediately. If you have other symptoms that are not severe, you can discuss them (virtually or in-person) with your primary healthcare professional. You should tell the health care providers who see you that you received the AstraZeneca/COVISHIELD or Janssen/Johnson \& Johnson COVID-19 vaccine and give them the date you got vaccinated. If the healthcare professional who assesses you is concerned, you may have scans done and additional bloodwork collected. When recognized early, VITT can be successfully treated.

\section{Do healthcare professionals know how to diagnose and treat VITT?}

Health care professionals and scientists in Ontario have been working with experts in Canada, and around the world, to better understand VITT. The Ontario COVID-19 Science Advisory Table has summarized what we know about VITT right now and has published guides for healthcare professionals outside and inside of hospitals to help them diagnose and treat VITT.

\section{Why is Ontario still using the AstraZeneca/COVISHIELD and Janssen/Johnson \& Johnson COVID-19 vaccines?}

Health Canada has authorized the AstraZeneca/COVISHIELD COVID-19 vaccine, as well as the Janssen/Johnson \& Johnson vaccine for use in Canadians aged 18 years and above. The National Advisory Committee on Immunization (NACl) makes a strong preferential recommendation for mRNA vaccines for all Canadians. $\mathrm{NACl}$ has recommended that the AstraZeneca/COVISHIELD and Janssen/Johnson \& Johnson vaccine may be offered to Canadians 30 years of age and older, if the benefits outweigh risks of waiting for an mRNA vaccine, the decision to receive the vaccine is informed by risks and consequences of VITT, and the delay to receive an mRNA vaccine is substantial.

There have been over 1.1 million cases of COVID-19 diagnosed in Canada so far, COVID-19 has killed over 24,000 Canadians, about 1 in 100 Canadians who get COVID-19 end up needing intensive care. The AstraZeneca/COVISHIELD and Janssen/ Johnson \& Johnson vaccines are both effective at reducing hospitalization and severe illness from COVID-19, and at reducing the chance you could pass the SARSCoV-2 virus on to others. Health care professionals, scientists, and government agencies in Ontario - and around the world - will continue to monitor the safety of these and all COVID-19 vaccines. 


\section{Could other COVID-19 vaccines available in Ontario cause VITT?}

There have been no confirmed cases of VITT with the Pfizer or Moderna COVID-19 vaccines. 